\title{
MicroRNA-203 inhibits the malignant progression of neuroblastoma by targeting Sam68
}

\author{
DONGJU ZHAO ${ }^{1}$, YUNJIAO TIAN ${ }^{1}$, PEILING LI ${ }^{1}$, LIMIN WANG $^{1}$, \\ AIJU XIAO ${ }^{1}$, MINGQIU ZHANG ${ }^{2}$ and TAIXIN SHI ${ }^{1}$ \\ Departments of ${ }^{1}$ Pediatrics and ${ }^{2}$ Interventional Radiography, The First Affiliated Hospital of Xinxiang Medical University, \\ Weihui, Henan 453100, P.R. China
}

Received October 14, 2014; Accepted June 15, 2015

DOI: $10.3892 / \mathrm{mmr} .2015 .4013$

\begin{abstract}
Neuroblastoma (NB) is the most common solid extracranial tumor in children. However, the molecular mechanism of NB remains to be elucidated. In the present study, reverse transcription quantitative polymerase chain reaction data demonstrated that the expression of Sam68 was significantly upregulated in NB tissues compared with their matched adjacent normal tissues. Furthermore, it was revealed that reduced expression of miR-203 and increased expression of Sam68 co-existed in NB tissues. Knockdown of Sam68 reduced the proliferation, migration and invasion of human SK-N-SH and SH-SY5Y NB cells. Similarly, overexpression of miR-203 also inhibited the proliferation, migration and invasion of these two cell lines. It was further demonstrated that the protein expression level of Sam68 was negatively mediated by miR-203 in the SK-N-SH and SH-SY5Y NB cells. Additionally, data from a dual luciferase reporter assay confirmed that miR-203 directly targeted Sam68 by binding to its 3'-untranslated region. In conclusion, the present study suggested for the first time, to the best of our knowledge, that the aberrant downregulation of miR-203 may contribute to the aberrant upregulation of Sam68 in NB and that miR-203 has an inhibitory role in malignant progression of NB by targeting Sam68. The present study provided evidence to support miR-203/Sam68 as a novel diagnostic or therapeutic targets for NB.
\end{abstract}

\section{Introduction}

Neuroblastoma (NB) is the most common solid extracranial tumor in children, accounting for $15 \%$ of all childhood cancer-associated mortalities. In spite of significant advances

Correspondence to: Dr Dongju Zhao, Department of Pediatrics, The First Affiliated Hospital of Xinxiang Medical University, 88 Jiankang Road, Weihui, Henan 453100, P.R. China

E-mail: xinxiangzhaodongju@163.com

Key words: neuroblastoma, microRNA-203, Sam68, malignant progression in detection and effort to reduce recurrence rates, the prognosis of NB remains poor. The predominant obstacle of NB therapy is recurrence, which is characterized by metastasis $(1,2)$. Therefore, it is urgent to identify the molecular mechanism involved in NB metastasis.

MicroRNAs (miRNAs) regulate gene expression by inhibiting gene translation or facilitating mRNA degradation (3). In previous years, accumulating evidence has revealed that miRNAs modulate a variety of genes pivotal for tumor development (4). Deregulation of miRNAs contribute to abnormal expression of oncogenes and tumor suppressors. Previously, miRNA (miR)-203 has been reported to be involved in the development of certain types of cancer. However, the detailed role of miR-203 in NB, as well as the underlying molecular mechanism, remain to be elucidated.

Sam68 is a member of the $\mathrm{K}$ homology domain-containing, RNA-binding, signal transduction-associated protein family, and has been suggested to be involved in various cellular processes, including RNA 3'-end formation, alternative splicing, cell cycle regulation, adipogenesis, neuronal development and tumorigenesis (5-7). Previously, Sam68 was identified to be frequently upregulated in cancer and has an oncogenic role in inflammatory carcinogenesis, tumor invasion and metastasis $(8,9)$. Sam68 is predicated to be a target gene of miR-203, according to bioinformatic analysis, however, whether miR-203 directly mediates the expression of Sam68 and their roles in NB remain to be elucidated.

In the present study, the expression levels of Sam68 and miR-203 were determined in NB tissues and adjacent normal tissues. The roles of Sam68 and miR-203 in the regulation of cell proliferation, migration and invasion of human SK-N-SH and SH-SY5Y NB cell lines were further assessed. In addition, the targeting association between miR-203 and Sam68 was determined in SK-N-SH and SH-SY5Y cells.

\section{Materials and methods}

Reagents. TRIzol reagent, Dulbecco's modified Eagle's medium (DMEM), fetal bovine serum (FBS), Lipofectamine 2000, Opti-MEM medium and an mirVana ${ }^{\mathrm{TM}}$ qRT-PCR microRNA Detection kit were purchased from Life Technologies (Carlsbad, CA, USA). A standard SYBR Green RT-PCR kit was purchased from Takara (Otsu, Japan). Sam68 siRNA, 
miR-203 mimics and scramble miRNA were purchased from Genecopoeia (Guangzhou, China). A Bradford DC protein assay kit was purchased from Bio-Rad Laboratories, Inc. (Hercules, CA, USA). Mouse anti-Sam68 (cat. no. sc-1238) and mouse anti-GAPDH (sc-59540) antibodies, and rabbit anti-mouse secondary antibody (cat. no. sc-358943) were purchased from Santa Cruz Biotechnology, Inc. (Dallas, TX, USA). An enhanced chemiluminescence (ECL) kit was purchased from Pierce Chemical (Rockford, IL, USA). A cell invasion assay kit was purchased from Chemicon (Temecula, CA, USA) and a Quick-Change Site-Directed Mutagenesis kit was purchased from Stratagene (La Jolla, CA, USA). The PsiCHECK ${ }^{\mathrm{TM}} 2$ vector and Dual-Glo substrate system was purchased from Promega (Madison, WI, USA).

Tissue specimen collection and cell culture. The present study was approved by the Ethics Committee of Xinxiang Medical University (Xinxiang, China). A total of 16 NB tissues and their matched normal adjacent tissues were collected at the Department of Pediatrics, The First Affiliated Hospital of Xinxiang Medical University (Weihui, China). Informed consent was obtained from the parents/guardians of the patients. Human SK-N-SH and SH-SY5Y NB cell lines were obtained from China Center for Type Culture Collection (Wuhan, China). The cells were cultured in DMEM, supplemented with $10 \% \mathrm{FBS}, 100 \mathrm{IU} / \mathrm{ml}$ penicillin and $100 \mu \mathrm{g} / \mathrm{ml}$ streptomycin sulfate (Life Technologies) at $37^{\circ} \mathrm{C}$ in a humidified incubator, containing $5 \% \mathrm{CO}_{2}$.

Reverse transcription quantitative polymerase chain reaction (RT-qPCR). The total RNA was extracted from cells using TRIzol reagent. The relative expression of miR-203 was determined by RT-qPCR using a mirVana ${ }^{\mathrm{TM}}$ qRT-PCR microRNA Detection kit, according to the manufacturer's instructions. The mRNA expression of Sam68 was detected by RT-qPCR using the standard SYBR Green RT-PCR kit, according to the manufacturer's instructions. GAPDH was used as an internal control. The specific primer pairs (Genecopoeia) were as follows: Sam68, sense: 5'-ATTCTTGGACCACAAGGGAATAC-3' and antisense: 5'-GCCATAAGAGCATAAGCCTCACA-3'; GAPDH, sense: 5'-GGAGCGAGATCCCTCCAAAAT-3' and antisense: 5'-GGCTGTTGTCATACTTCTCATGG-3'. The relative mRNA expression levels of Sam68 or miR-203 were quantified using the GraphPad Prism 4.0 software (GraphPad Software, San Diego, CA, USA) and the $2^{-\Delta \Delta C t}$ method (10).

Transfection. Transfection was performed using Lipofectamine 2000, according to the manufacturer's instructions. At the time of transfection, the cells were at least $70 \%$ confluent. Oligonucleotides and plasmids were incubated in Opti-MEM medium at $37^{\circ} \mathrm{C}$ for $20 \mathrm{~min}$. For miR-148a functional analysis, the cells were transfected with $100 \mathrm{nM}$ pre-miR-203, anti-miR-203, pre-miR-con and anti-miR-con. For Sam68 functional analysis, the cells were transfected with Sam68-specific siRNA.

Cell proliferation assay. For each group, 10,000 cells/well were seeded into a 96 -well plate. Following transfection, the plates were incubated for $48 \mathrm{~h}$ at $37^{\circ} \mathrm{C}$ with $5 \% \mathrm{CO}_{2}$. To assess cell proliferation, an MTT assay was performed, according to the manufacturer's instructions. MTT reagent $(10 \mu \mathrm{l} ; 5 \mathrm{mg} / \mathrm{ml}$; Life Technologies) in phosphate-buffered saline was added to each well and incubated for $4 \mathrm{~h}$ at $37^{\circ} \mathrm{C}$ with $5 \% \mathrm{CO}_{2}$. The supernatant was subsequently removed and $100 \mu \mathrm{l}$ dimethyl sulfoxide was added. The absorbance was measured at 490 nm using a Microplate Reader (Model 680; Bio-Rad Laboratories, Inc.). Each assay was performed in triplicate wells and repeated three times.

Cell migration assay. Cell migration was measured using a wound healing assay. Briefly, the cells were cultured to confluence. Wounds of $\sim 1 \mathrm{~mm}$ width were created with a plastic tip. Following wounding, the cells were incubated for $24 \mathrm{~h}$ and were subsequently fixed with $16 \%$ trichloroacetic acid and observed under a microscope (IX83; Olympus Corporation, Tokyo, Japan).

Cell invasion assay. Cell invasion was measured using a Cell Invasion Assay kit, according to the manufacturer's instruction. Briefly, the cells in serum-free DMEM were seeded into the upper compartment of the chambers and DMEM, containing $10 \% \mathrm{FBS}$, was added into the lower chambers. Following incubation for $24 \mathrm{~h}$ at $37^{\circ} \mathrm{C}$, the cells on the upper face of the membrane were removed using a cotton swab and the cells on the lower face were fixed with $16 \%$ trichloroacetic acid, stained with $0.1 \%$ crystal violet (Life Technologies) and observed under a microscope (IX83; Olympus Corporation).

Western blotting. The total protein was extracted using the Tissue or Cell Protein Extraction kit (Amresco, Beijing, China) and the protein concentration was measured using the Bradford DC protein assay kit. The proteins were separated by $10 \%$ SDS-PAGE (Pierce Chemical) and blotted onto polyvinylidene difluoride membranes (PVDF; Pierce Chemical), which were subsequently blocked with Tris-buffered saline with $0.5 \%$ Tween-20, containing $50 \mathrm{~g} / 1$ non-fat milk, at room temperature for $4 \mathrm{~h}$. Following incubation, the PVDF membrane was incubated with the primary antibodies, anti-Sam68 and anti-GAPDH (1:200 dilution), at $37^{\circ} \mathrm{C}$ for $1 \mathrm{~h}$. The membranes were rinsed with Dulbecco's phosphate-buffered saline (DPBS; Life Technologies) and incubated for $1 \mathrm{~h}$ with the corresponding peroxidase-conjugated rabbit anti-mouse secondary antibody (1:20,000 dilution). The membranes were further washed with DPBS and chemiluminescent detection was performed using the ECL kit.

Dual luciferase reporter assay. The SH-SY5Y cells were co-transfected with the reporter constructs, Sam68-3'untranslated region (UTR)-psi-CHECK2 (containing the 3'-UTR of Sam68 and the miRNA-203 binding sites) or the mutated (Mut)-Sam68-3'UTR-psi-CHECK2 (containing the corresponding mutated sequence of 3'-UTR of Sam68), and the miR-203 mimics or scramble miRNA as a negative control, using Lipofectamine 2000. The luciferase activity was determined after $48 \mathrm{~h}$ using the Dual-Glo substrate system and LD400 luminometer (Beckman Coulter, Kraemer Boulevard Brea, CA, USA). The data are presented as the ratio of Renilla luciferase to Firefly luciferase. 
A

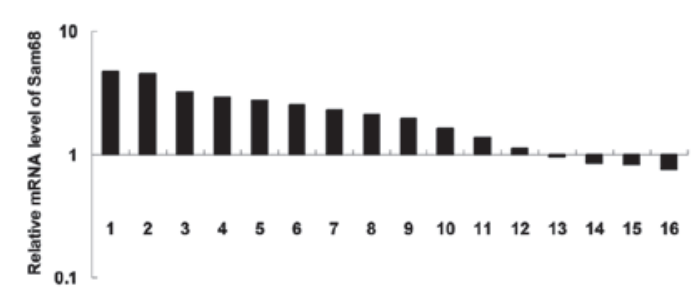

C

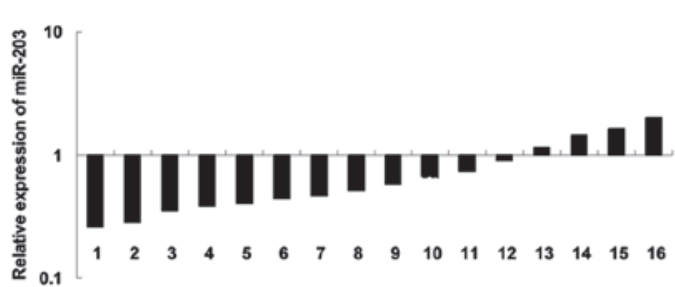

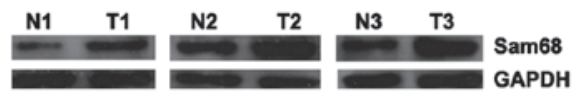

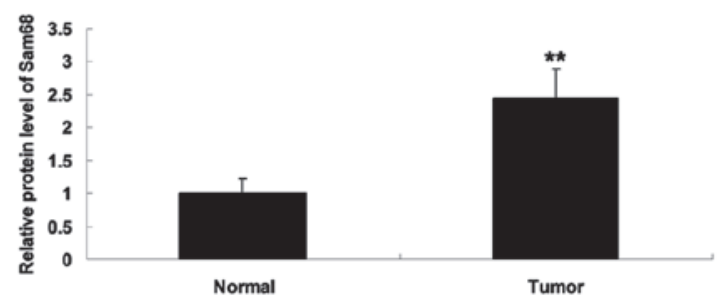

D

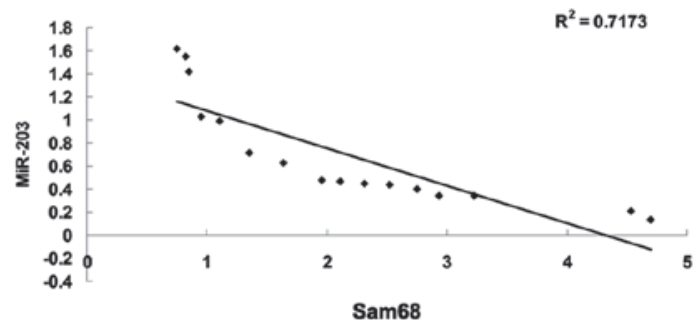

Figure 1. (A) Reverse transcription quantitative polymerase chain reaction was performed to determine the relative mRNA expression levels of Sam68 in the 16 NB tissues compared with their matched adjacent normal tissues. GAPDH was used as an internal control. (B) A western blot assay was performed to determine the relative protein expression levels of Sam68 in 16 NB tissues compared with their matched adjacent normal tissues. GAPDH was used as an internal loading control. The western blotting data from three representative patients with NB are shown $\left({ }^{* *} \mathrm{P}<0.01\right.$, vs. Normal). (C) Reverse transcription quantitative polymerase chain reaction was performed to determine the relative expression levels of miR-203 in the $16 \mathrm{NB}$ tissues compared with their matched adjacent normal tissues. U6 was used as an internal control. (D) An inverse correlation between the expression levels of miR-203 and Sam68 was observed (R2=0.7173). NB, neuroblastoma; N, normal; T, tumor.

Statistical analysis. The data are expressed as the mean \pm standard deviation. Differences between the two groups were determined by Student t-test. All analyses were performed using SPSS 17.0 software (IBM SPSS, Chicago, IL, USA). $\mathrm{P}<0.05$ was considered to indicate a statistically significant difference.

\section{Results}

Inverse expression levels of Sam68 and miR-203 in NB tissues. The present study investigated the expression of Sam68 in NB tissues. As shown in Fig. 1A and B, the mRNA and protein expression levels of Sam68 were frequently upregulated in the NB tissues compared with their matched adjacent normal tissues. It was further demonstrated that the expression level of miR-203 was frequently reduced in the NB tissues compared with their matched normal adjacent tissues (Fig. 1C). Notably, an inverse correlation between the expression levels of miR-203 and Sam68 were observed $\left(R^{2}=0.7173\right.$, Fig. 1D). Therefore, it was suggested that deregulation of Sam68 and miR-203 may be important in NB.

Knockdown of Sam68 inhibits the proliferation, migration and invasion of NB cells. To investigate the role of Sam68 in NB, the SK-N-SH and SH-SY5Y cells were transfected with Sam68-specific siRNA. As shown in Fig. 2A and B, the mRNA and protein expression levels of Sam68 were significantly decreased in the SK-N-SH and SH-SY5Y cells following transfection with Sam68-specific siRNA. It was also demonstrated that the downregulation of Sam68 inhibited the proliferation, migration and invasion of the SK-N-SH and SH-SY5Y cells (Fig. 2C-E). These findings suggested that Sam68 may be involved in the progression of NB.
Overexpression of miR-203 suppresses the proliferation, migration and invasion of $N B$ cells. The present study further assessed the role of miR-203 in NB. Since miR-203 was observed to be downregulated in NB, the SK-N-SH and SH-SY5Y cells were transfected with pre-miR-203 plasmid. As shown in Fig. 3A, transfection with pre-miR-203 significantly increased the expression levels of miR-203 in the SK-N-SH and SH-SY5Y cells. It was demonstrated that the upregulation of miR-203 markedly suppressed proliferation, migration and invasion of the SK-N-SH and SH-SY5Y cells (Fig. 3B-D). These findings suggested that miR-203 functions as a tumor suppressor in NB.

miR-203 negatively regulates the expression of Sam68 by directly binding to its 3'-UTR. The effects of the upregulation of miR-203 on the expression of Sam68 were further assessed. The SK-N-SH and SH-SY5Y cells were transfected with miR-203 mimics and the protein expression of Sam68 was determined by western blotting. As shown in Fig. 4A, overexpression of miR-203 significantly inhibited the protein expression of Sam68 in the SK-N-SH and SH-SY5Y cells. These data indicated that miR-203 negatively mediated the expression of Sam68 in NB cells.

Whether Sam68 is a direct target gene of miR-203 was next investigated. The Sam68 3'-UTR, containing the wild type (WT) or mutant type (Mut) of the miR-203 binding site, was subcloned into psi-CHECK2 dual luciferase reporter vectors (Fig. 4B). As shown in Fig. 4C, overexpression of miR-203 markedly decreased the luciferase activity in the SH-SY5Y cells transfected with the WT Sam68-3'UTR-psi-CHECK2 reporter vector, however, the luciferase activity in the SH-SY5Y cells co-transfected with the Mut-Sam68-3'UTR-psi-CHECK2 rep orter vector and miR-203 exhibited no difference compared 
A

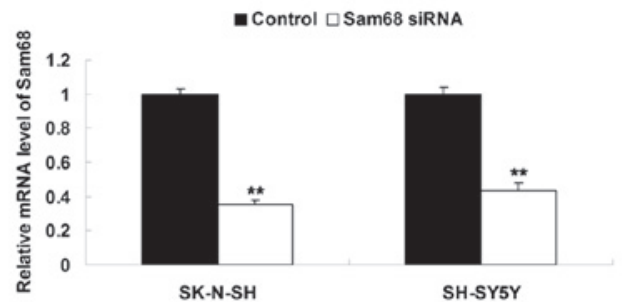

C

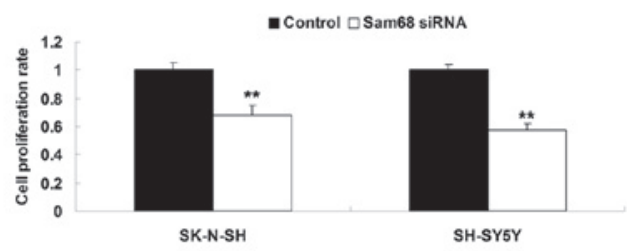

D

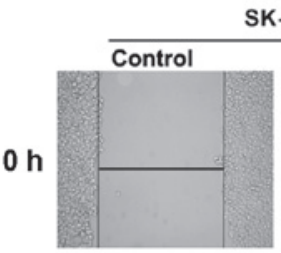

SK-N-SH

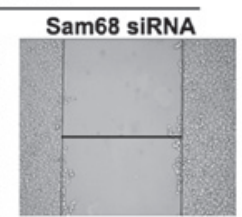

$24 \mathrm{~h}$
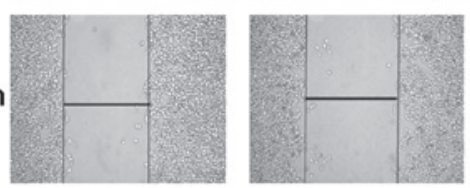

$\mathbf{E}$

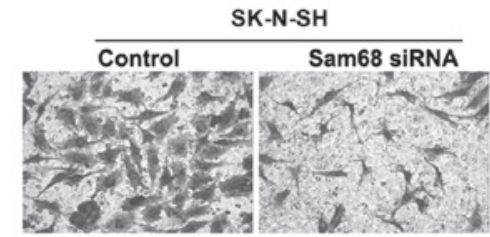

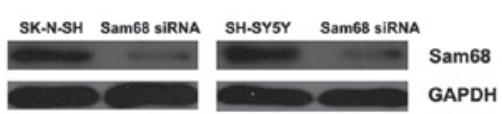
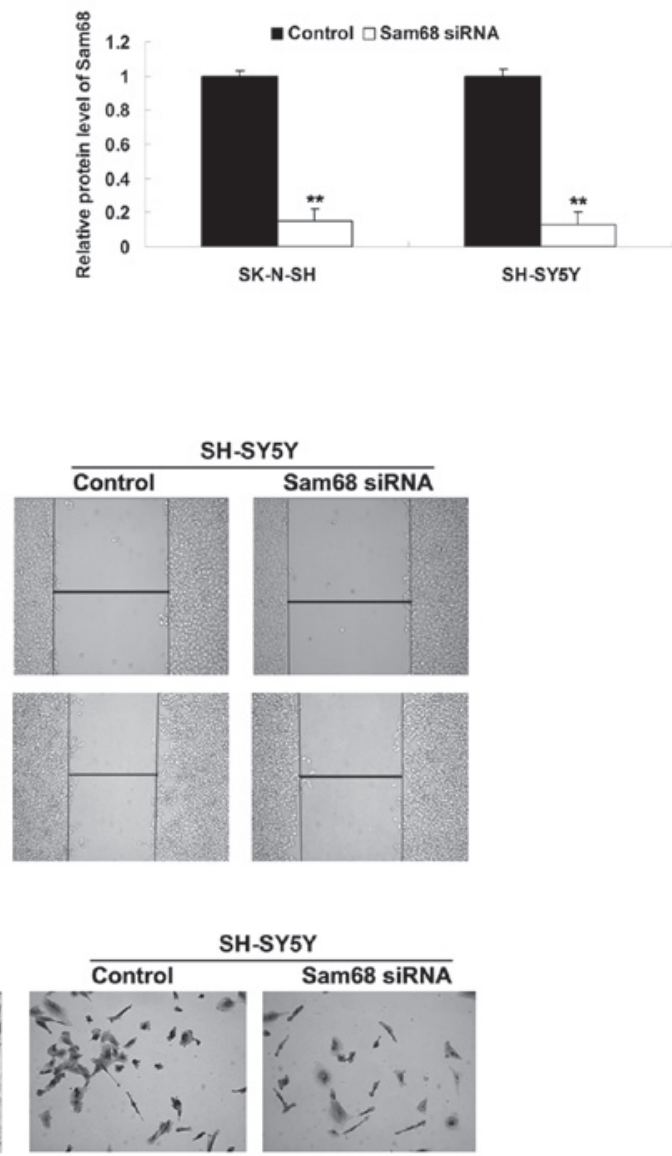

Figure 2. (A) Reverse transcription quantitative polymerase chain reaction was performed to determine the relative mRNA expression level of Sam68 in the two NB cell lines transfected with Sam68-specific siRNA. GAPDH was used as an internal control ("* $\mathrm{P}<0.01$, vs. Control). (B) A western blot assay was performed to determine the relative protein expression level of Sam68 in the two NB cell lines transfected with Sam68-specific siRNA. GAPDH was used as an internal loading control ( ${ }^{* *} \mathrm{P}<0.01$, vs. Control). (C) An MTT assay was performed to determine the cell proliferation of the two NB cell lines transfected with Sam68-specific siRNA (*** $\mathrm{P}<0.01$, vs. Control). (D) A scratch assay was performed to determine the migratory capacity of the two NB cell lines transfected with Sam68-specific siRNA (magnification, x100). (E) A Transwell assay was performed to determine the invasive capacity of the two NB cell lines transfected with Sam68-specific siRNA (magnification, x100). Control, cells without transfection; NB, neuroblastoma.

with the control group (Fig. 4D). This indicated that Sam68 is a novel target of miR-203. Based on these findings, the upregulation of Sam68 may be induced partly by the downregulation of miR-203 and miR-203 may act as a tumor suppressor in NB by targeting Sam68.

\section{Discussion}

Sam68 localizes in the nucleus and is involved in the formation of both nuclear and cytosolic multi-molecular complexes, including stress granules and Sam68 nuclear bodies (11). In addition, Sam68 mediates gene expression by coupling with other proteins and RNA targets, and therefore, is involved in the regulation of certain important cellular functions, including cell proliferation, differentiation and death (11). For instance, Sam68 was recently identified to modulate the promoter specificity of $\mathrm{NF}-\kappa \mathrm{B}$ and regulate the expression of CD25 in activated T cells (12).
Deregulation of oncogenes or tumor suppressors is critical in the initiation and progression of cancer. It has been reported that high expression of Sam68 is a is a predictor of poor prognosis in non-small cell lung cancer, oral tongue cancer, early-stage cervical cancer and colorectal cancer $(8,9,13,14)$. In the present study, the expression level of Sam68 was demonstrated to be higher in NB tissues compared with their matched adjacent normal tissues. It was also demonstrated that the knockdown of Sam68 effectively inhibited the proliferation, migration and invasion of the SK-N-SH and SH-SY5Y NB cells. Previously, Zhao et al (15) also reported that Sam68 was upregulated in NB tissues and cell lines. Furthermore, the authors revealed that the protein expression of Sam68 was positively correlated with clinical stage, tumor histology and distant metastasis, and that high expression of Sam68 was a predictor of poor prognosis of patients with NB (15). Taken together, the present study suggested that the deregulation of Sam68 may be involved in the development and progression of NB. 
A

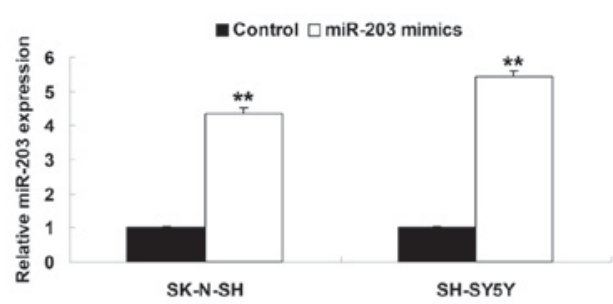

C

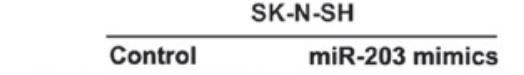

$\mathrm{Oh}$

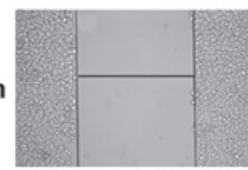

$24 \mathrm{~h}$
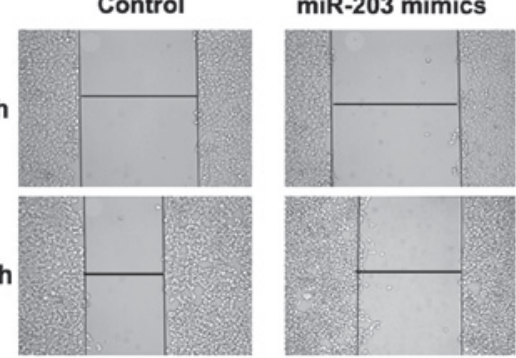

D

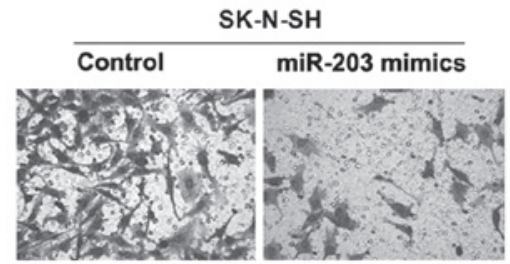

B
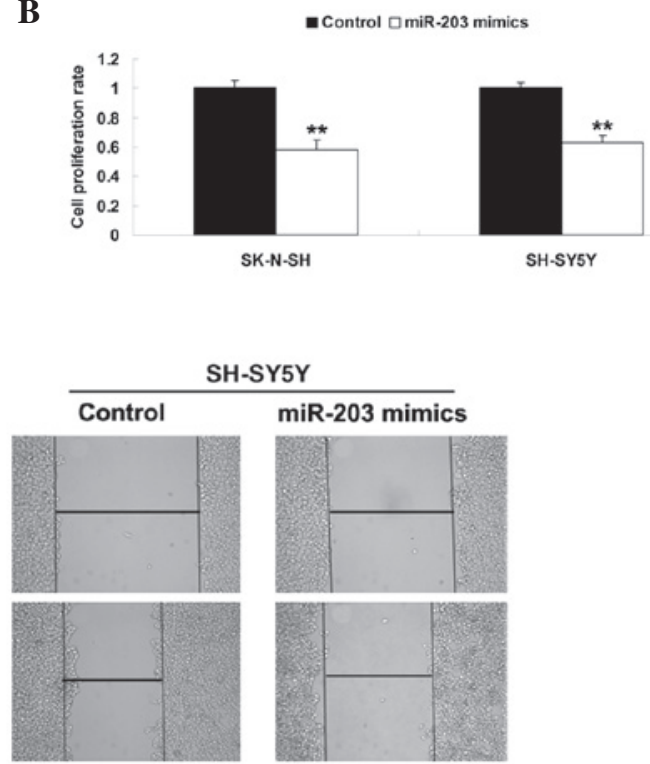

SH-SY5Y

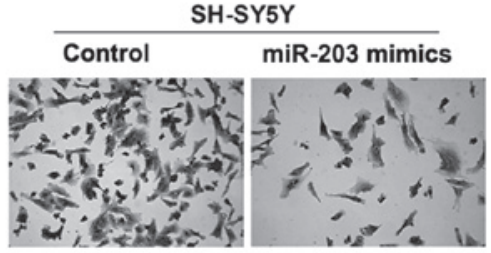

Figure 3. (A) Reverse transcription quantitative polymerase chain reaction was performed to examine the relative expression level of miR-203 in the two NB cell lines transfected with the miR-203 mimics. GAPDH was used as an internal control ( ${ }^{* *} \mathrm{P}<0.01$, vs. Control). (B) An MTT assay was performed to determine the cell proliferation of the two NB cell lines transfected with the miR-203 mimics ( $" * \mathrm{P}<0.01$, vs. Control). (C) A scratch assay was performed to determine the migratory capacity of the two NB cell lines transfected with the miR-203 mimics (magnification, x100). (D) A Transwell assay was performed to determine the invasive capacity of the two NB cell lines transfected with the miR-203 mimics (magnification, x100). Control, cells without transfection; NB, neuroblastoma.

A

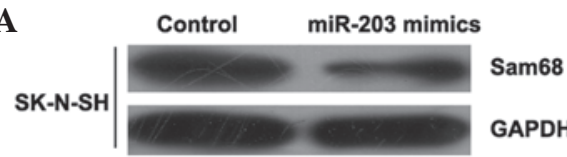

SH-SY5Y

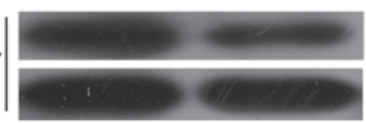

- Control $\square$ miR-203 mimics

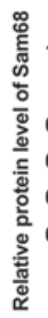

GAPDH
B

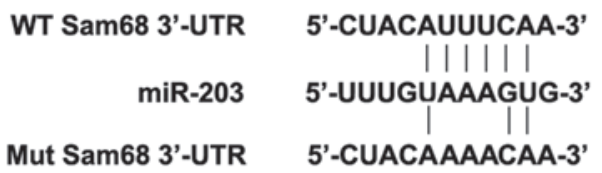

$\mathbf{C}$

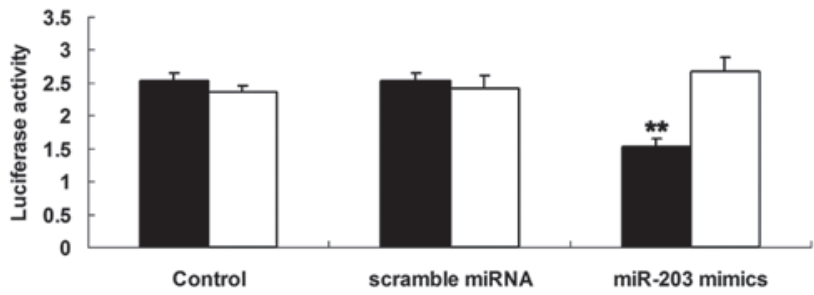

Figure 4. (A) Western blotting was performed to determine the relative protein expression level of Sam68 in the two NB cell lines transfected with the miR-203 mimics. GAPDH was used as an internal control ( ${ }^{* *} \mathrm{P}<0.01$, vs. Control). (B) The sequences for miR-203 at the WT or Mut 3'UTR of Sam68 are shown. (C) A luciferase reporter assay was performed to confirm whether Sam68 is a target gene of miR-203. The luciferase activity was reduced only in the SH-SY5Y cells co-transfected with the miR-203 mimics and WT Sam68 3'UTR, however, not in the SH-SY5Y cells co-transfected with the miR-203 mimics and Mut Sam68 3'UTR ( ${ }^{* *} \mathrm{P}<0.01$, vs. Control). Control, cells without transfection; NB, neuroblastoma; WT, wild-type; Mut, mutant; UTR, untranslated region.

The regulatory mechanisms of oncogenes, including methylation, transcription factors and mutation, are complicated. Previously, accumulating evidence has demonstrated that the deregulation of oncogenes or tumor suppressors is due to the aberrant expression of its regulatory miRNAs. However, the molecular mechanism of the expression of Sam68 in NB 
remains to be elucidated. In the present study, the expression of miR-203, a theoretical regulatory miRNA of Sam68 predicted by algorithms, was assessed in NB tissues and cell lines. The data revealed that the expression level of miR-203 was significantly reduced in NB tissues compared with their matched adjacent normal tissues, suggesting that the aberrant downregulation of miR-203 may be involved in the development and progression of NB. In fact, deregulation of miR-203 has been demonstrated to be associated with various cancer types, including hepatocellular carcinoma, pancreatic cancer, glioma, lung cancer, cervical cancer, prostate carcinoma, melanoma, esophageal squamous cell carcinoma and colon cancer (16-25).

The present study further revealed that the overexpression of miR-203 markedly suppressed the proliferation, migration and invasion of the SK-N-SH and SH-SY5Y NB cells, similar to the effects of Sam68 downregulation. In addition, the present study demonstrated for the first time, to the best of our knowledge, that Sam68 is a novel target of miR-203, and that miR-203 and these miRNAs negatively regulated the expression of Sam68 by targeting its 3'-UTR in NB cells. Therefore, it is possible that high expression of Sam68 in NB is partly due to the downregulation of the expression of miR-203. The suppressive role of miR-203 in human cancer has been widely investigated. For instance, Tian et al (26) demonstrated that the expression of miR-203 was significantly reduced in laryngeal squamous cell carcinoma tissues, and downregulation of miR-203 was correlated with advanced clinical stages, lymph node metastasis and poor prognosis (26).

An miRNA has various target genes and a gene can be regulated by multiple miRNAs. It has been demonstrated that miR-203 can target survivin, leading to cell cycle arrest in laryngeal carcinoma cells (27). It can also target Hakai and inhibit the growth of human colon adenocarcinoma (28). Additionally, those genes involved in the regulation of cell proliferation, apoptosis, cell cycle progression, migration and invasion, are also the targets of miR-203, including Robo1, Kif5b, E2F3, LASP1, ASAP1, PKC $\alpha$, BIRC5, Bmi-1 and DeltaNp63 (23-26,29-33). Whether these miR-203 targets are also involved in the development and progression of NB remains to be elucidated.

In conclusion, the present study demonstrated that Sam68 is upregulated in NB tissues and cell lines, which is likely due to the downregulation of the expression of miR-203. In addition, the overexpression of miR-203 effectively inhibited the proliferation, migration and invasion of NB cell lines, partly at least, by directly targeting Sam68.

\section{References}

1. Fulda S: The PI3K/Akt/mTOR pathway as therapeutic target in neuroblastoma. Curr Cancer Drug Targets 9: 729-737, 2009.

2. Jrebi NY, Iqbal CW, Joliat GR, Sebo TJ and Farley DR: Review of our experience with neuroblastoma and ganglioneuroblastoma in adults. World J Surg, 2871-2874, 2014.

3. Ambros V: The functions of animal microRNAs. Nature 431: 350-355, 2004.

4. Baer C, Claus R and Plass C: Genome-wide epigenetic regulation of miRNAs in cancer. Cancer Res 73: 473-477, 2013.

5. Vogel G and Richard S: Emerging roles for Sam68 in adipogenesis and neuronal development. RNA Biol 9: 1129-1133, 2012.

6. Najib S, Martin-Romero C, Gonzalez-Yanes C and Sanchez-Margalet V: Role of Sam68 as an adaptor protein in signal transduction. Cell Mol Life Sci 62: 36-43, 2005.

7. Lukong KE and Richard S: Sam68, the KH domain-containing superSTAR. Biochim Biophys Acta 1653: 73-86, 2003.
8. Chen SW, Zhang Q, Yang AK, Li Z, Zhong Y, Li H, Zeng Y, Zhuang SM, Wang LP, Song LB, et al: Overexpression and cytoplasmic localization of Sam68 correlate with tumour progression and poor prognosis in patients with clinically N0 oral tongue cancer. Head Neck Oncol 4: 61, 2012.

9. Zhang Z, Xu Y, Sun N, Zhang M, Xie J and Jiang Z: High Sam68 expression predicts poor prognosis in non-small cell lung cancer. Clin Transl Oncol 16: 886-891, 2014.

10. Arocho A, Chen B, Ladanyi M and Pan Q: Validation of the 2-DeltaDeltaCt calculation as an alternate method of data analysis for quantitative PCR of BCR-ABL P210 transcripts. Diagn mol pathol 15: 56-61, 2006.

11. Sánchez-Jiménez F and Sánchez-Margalet V: Role of Sam68 in post-transcriptional gene regulation. Int J Mol Sci 14: 23402-23419, 2013.

12. Fu K, Sun X, Zheng W, Wier EM, Hodgson A, Tran DQ, Richard S and Wan F: Sam68 modulates the promoter specificity of NF-kB and mediates expression of CD25 in activated T cells. Nat Commun 4: 1909, 2013

13. Liao WT, Liu JL, Wang ZG, Cui YM, Shi L, Li TT, Zhao XH, Chen XT, Ding YQ and Song LB: High expression level and nuclear localization of Sam68 are associated with progression and poor prognosis in colorectal cancer. BMC Gastroenterol 14: $126,2014$.

14. Li Z, Yu CP, Zhong Y, Liu TJ, Huang QD, Zhao XH, Huang H, Tu H, Jiang S, Zhang Y, et al: Sam68 expression and cytoplasmic localization is correlated with lymph node metastasis as well as prognosis in patients with early-stage cervical cancer. Ann Oncol 23: 638-646, 2012.

15. Zhao X, Li Z, He B, Liu J, Li S, Zhou L, Pan C, Yu Z and Xu Z: Sam68 is a novel marker for aggressive neuroblastoma. Onco Targets Ther 6: 1751-1760, 2013.

16. Furuta M, Kozaki KI, Tanaka S, Arii S, Imoto I and Inazawa J: miR-124 and miR-203 are epigenetically silenced tumor-suppressive microRNAs in hepatocellular carcinoma. Carcinogenesis 31: 766-776, 2010.

17. Greither T, Grochola LF, Udelnow A, Lautenschlager C, Wurl $P$ and Taubert $H$ : Elevated expression of microRNAs 155, 203, 210 and 222 in pancreatic tumors is associated with poorer survival. Int J Cancer 126: 73-80, 2010.

18. He J, Deng Y, Yang G and Xie W: MicroRNA-203 down-regulation is associated with unfavorable prognosis in human glioma. J Surg Oncol, 108: 121-125, 2013.

19. Cheung TH, Man KN, Yu MY, Yim SF, Siu NS, Lo KW, Doran G, Wong RR, Wang VW, Smith DI, et al: Dysregulated microRNAs in the pathogenesis and progression of cervical neoplasm. Cell Cycle 11: 2876-2884, 2012.

20. Boll K, Reiche K, Kasack K, Mörbt N, Kretzschmar AK, Tomm JM, Verhaegh G, Schalken J, von Bergen M, Horn F, et al: MiR-130a, miR-203 and miR-205 jointly repress key oncogenic pathways and are downregulated in prostate carcinoma. Oncogene 32: 277-285, 2013.

21. Kozubek J, Ma Z, Fleming E, Duggan T, Wu R, Shin DG and Dadras SS: In-depth characterization of microRNA transcriptome in melanoma. PLoS One 8: e72699, 2013.

22. Schetter AJ, Leung SY, Sohn JJ, Zanetti KA, Bowman ED, Yanaihara N, Yuen ST, Chan TL, Kwong DL, Au GK, et al: MicroRNA expression profiles associated with prognosis and therapeutic outcome in colon adenocarcinoma. JAMA 299: 425-436, 2008.

23. Wang C, Wang X, Liang H, Wang T, Yan X, Cao M, Wang N, Zhang S, Zen K, Zhang C, et al: miR-203 Inhibits cell proliferation and migration of lung cancer cells by targeting PKC $\alpha$. PLoS One 8: e73985, 2013.

24. Wang C, Zheng X, Shen C and Shi Y: MicroRNA-203 suppresses cell proliferation and migration by targeting BIRC5 and LASP1 in human triple-negative breast cancer cells. J Exp Clin Cancer Res 31: 58, 2012.

25. Yuan Y, Zeng ZY, Liu XH, Gong DJ, Tao J, Cheng HZ and Huang SD: MicroRNA-203 inhibits cell proliferation by repressing $\triangle \mathrm{Np} 63$ expression in human esophageal squamous cell carcinoma. BMC Cancer 11: 57, 2011.

26. Tian L, Li M, Ge J, Guo Y, Sun Y, Liu M and Xiao H: MiR-203 is downregulated in laryngeal squamous cell carcinoma and can suppress proliferation and induce apoptosis of tumours. Tumour Biol 35: 5953-5963, 2014.

27. Bian K, Fan J, Zhang X, Yang XW, Zhu HY, Wang L, Sun JY, Meng YL, Cui PC, Cheng SY, et al: MicroRNA-203 leads to G1 phase cell cycle arrest in laryngeal carcinoma cells by directly targeting survivin. FEBS Lett 586: 804-809, 2012. 
28. Abella V, Valladares M, Rodriguez T,Haz M, Blanco M, Tarrío N Iglesias P, Aparicio LA and Figueroa A: miR-203 regulates cell proliferation through its influence on Hakai expression. PLoS One 7: e52568, 2012.

29. Dontula R, Dinasarapu A, Chetty C, Pannuru P, Herbert E, Ozer $\mathrm{H}$ and Lakka SS: MicroRNA 203 modulates glioma cell migration via Robo1/ERK/MMP-9 Signaling. Genes Cancer 4 285-296, 2013.

30. Noguchi S, Kumazaki M, Yasui Y, Mori T, Yamada N and Akao Y: MicroRNA-203 regulates melanosome transport and tyrosinase expression in melanoma cells by targeting kinesin superfamily protein 5b. J Invest Dermatol, 134: 461-469, 2014.
31. Noguchi S, Mori T, Otsuka Y, Yamada N, Yasui Y, Iwasaki J, Kumazaki M, Maruo $\mathrm{K}$ and Akao Y: Anti-oncogenic microRNA-203 induces senescence by targeting E2F3 protein in human melanoma cells. J Biol Chem 287: 11769-11777, 2012.

32. Takeshita N, Mori M, Kano M, Hoshino I, Akutsu Y, Hanari N, Yoneyama Y, Ikeda N, Isozaki Y, Maruyama T, et al: miR-203 inhibits the migration and invasion of esophageal squamous cell carcinoma by regulating LASP1. Int J Oncol 41: 1653-1661, 2012.

33. Yu X, Jiang X, Li H, Guo L, Jiang W and Lu SH: MiR-203 inhibits the proliferation and self-renewal of esophageal cancer stem-like cells by suppressing stem renewal factor Bmi-1. Stem Cells Dev 23: 576-585, 2014. 